\title{
EXISTENCE OF INTEGRALS AND THE SOLUTION OF INTEGRAL EQUATIONS
}

BY

\author{
JON C. HELTON $\left.{ }^{1}\right)$
}

Abstract. Functions are from $R$ to $N$ or $R \times R$ to $N$, where $R$ denotes the real numbers and $N$ denotes a normed complete ring. If $S, T$ and $G$ are functions from $R \times R$ to $N$, each of $S\left(p^{-}, p\right), S\left(p^{-}, p^{-}\right), T\left(p^{-}, p\right)$ and $T\left(p^{-}, p^{-}\right)$exists for $a<p<b$, each of $S\left(p, p^{+}\right), S\left(p^{+}, p^{+}\right), T\left(p, p^{+}\right)$ and $T\left(p^{+}, p^{+}\right)$exists for $a<p<b, G$ has bounded variation on $[a, b]$ and $\int_{a}^{b} G$ exists, then each of

$$
\int_{a}^{b} S\left[G-\int G\right] T \text { and } \int_{a}^{b} S[1+G-\Pi(1+G)] T
$$

exists and is zero. These results can be used to solve integral equations without the existence of integrals of the form

$$
\int_{a}^{b}\left|G-\int G\right|=0 \text { and } \int_{a}^{b}|1+G-\Pi(1+G)|=0 .
$$

This is demonstrated by solving the linear integral equation

$$
f(x)=h(x)+(L R) \int_{a}^{x}(f G+f H)
$$

and the Riccati integral equations

$$
f(x)=w(x)+(L R L R) \int_{a}^{x}(f H+G f+f K f)
$$

without the existence of the previously mentioned integrals.

Introduction. The existence of integrals of the form

$$
\int_{a}^{b}\left|G-\int G\right|=0 \text { and } \int_{a}^{b}|1+G-\Pi(1+G)|=0
$$

can be very useful in studying the solution of integral equations and integral inequalities. This has been demonstrated in papers by B. W. Helton [7]-[12] and the author [15]. However, it is possible to construct functions such that the integrals in (i) do not exist. This has been done by W. D. L. Appling [2, Theorem 2, p. 155] and the author [13, pp. 153-154].

Presented in part to the Society, July 26, 1974 under the titles Existence of integrals and the solution of Riccati integral equations and Existence and approximation of integrals and February 3, 1975 under the title Product integrals and linear integral equations; received by the editors July 22, 1975 and, in revised form, December 14, 1975.

AMS (MOS) subject classifications (1970). Primary 45N05; Secondary 28A25, 26A39.

Key words and phrases. Sum integral, product integral, subdivision-refinement integral, existence, interval function, normed complete ring, linear integral equation, Riccati integral equation.

( ${ }^{1}$ This research was supported in part by a grant from Arizona State University. 
The purpose of this paper is to present results for use in solving integral equations in the event that the integrals in (i) do not exist. Two theorems of this nature are given. First, if $S, T$ and $G$ are functions from $R \times R$ to $N$, each of $S\left(p^{-}, p\right), S\left(p^{-}, p^{-}\right), T\left(p^{-}, p\right)$ and $T\left(p^{-}, p^{-}\right)$exists for $a<p<b$, each of $S\left(p, p^{+}\right), S\left(p^{+}, p^{+}\right), T\left(p, p^{+}\right)$and $T\left(p^{+}, p^{+}\right)$exists for $a<p<b$, $G$ has bounded variation on $[a, b]$ and $\int_{a}^{b} G$ exists, then $\int_{a}^{b} S\left[G-\int G\right] T$ exists and is zero. Second, if $S, T$ and $G$ are defined as before, then

$$
\int_{a}^{b} S[1+G-\Pi(1+G)] T
$$

exists and is zero. In the statement of these results, the requirement that $\int_{a}^{b} G$ exist can be replaced by the requirement that ${ }_{x} \Pi^{y}(1+G)$ exist for $a<x<y$ $<b$ (see Lemma 3).

To demonstrate the use of the results stated in the preceding paragraph, the linear integral equation

$$
f(x)=h(x)+(L R) \int_{a}^{x}(f G+f H)
$$

and the Riccati integral equation

$$
f(x)=w(x)+(L R L R) \int_{a}^{x}(f H+G f+f K f)
$$

are solved without requiring the existence of the integrals in (i). Further, the solutions are obtained with previous restrictions on $h$ and $w$ relaxed from bounded variation to quasi-continuity.

The solution presented for the linear integral equation in (ii) extends results by B. W. Helton [7, Theorem 5.1, p. 310] and the author [15, Theorem], [16, Theorem 1] since the integrals in (i) are not required to exist and $h$ is not required to have bounded variation. The ideas behind the existence results in the second paragraph are contained in a previous paper by the author [15], where the integral equation in (ii) is solved without requiring the existence of the integrals in (i). However, at the time, the nature of these results and their use in solving integral equations were not recognized. The presentation here is more direct than that in [15] and also more general since $h$ is not required to have bounded variation.

The solution presented for the Riccati integral equation in (iii) extends a result by B. W. Helton [11, Theorems 3.2, 3.3]. The result given here is more general since the integrals in (i) are not required to exist and $w$ is not required to have bounded variation.

This paper is part of a sequence of results initiated by two papers by $\mathbf{H}$. S. Wall [42]-[43]. There, in response to a paper by P. Puig Adam [1], Wall developed techniques involving continuous continued fractions and harmonic matrices for the solution of integral equations. In turn, these results have been extended in various directions by J. S. Mac Nerney [29]-[34], J. W. Neuberger 
[36]-[38], T. H. Hildebrandt [22], J. R. Dorroh [5], B. W. Helton [7]-[12], D. B. Hinton [23], J. V. Herod [18]-[21], C. W. Bitzer [3]-[4], D. L. Lovelady [24]-[28], J. A. Reneke [39]-[41], G. F. Webb [44]-[46], and the author [14]-[17]. For early background on product integration, the reader is referred to P. R. Masani [35] and F. R. Gantmacher [6, Chapter 24].

Definitions. All integrals and definitions are of the subdivision-refinement type, and functions are from $R$ to $N$ or $R \times R$ to $N$, where $R$ denotes the set of real numbers and $N$ denotes a ring which has a multiplicative identity element represented by 1 and a norm $|\cdot|$ with respect to which $N$ is complete and $|1|=1$. At times, we consider $2 \times 2$ and $1 \times 2$ matrices composed of elements of $N$. Then, we use $\max _{i=1,2} \Sigma_{j=1}^{2}\left|a_{i j}\right|$ and $\Sigma_{i=1}^{2}\left|a_{i}\right|$ as our norms and denote them by $\|\cdot\|$. Lower case letters are used to denote functions from $R$ to $N$, and capital letters are used to denote functions from $R \times R$ to $N$. Boldface letters are used to represent matrix functions. Functions on $R \times R$ are assumed to be defined only for elements $\{a, b\}$ of $R \times R$ such that $a<b$. If $\left\{x_{i}\right\}_{i=0}^{n}$ is a subdivision of $[a, b]$, then

$$
f_{i}=f\left(x_{i}\right) \text { and } G_{i}=G\left(x_{i-1}, x_{i}\right) .
$$

Further if $\left\{x_{i j}\right\}_{j=0}^{n(i)}$ is a subdivision of $\left[x_{i-1}, x_{i}\right]$, then $G_{i j}=G\left(x_{i, j-1}, x_{i j}\right)$ and $f_{i j}=f\left(x_{i j}\right)$.

The statement that $\int_{a}^{b} G$ exists means there exists an element $L$ of $N$ such that, if $\varepsilon>0$, then there exists a subdivision $D$ of $[a, b]$ such that, if $\left\{x_{i}\right\}_{i=0}^{n}$ is a refinement of $D$, then

$$
\left|L-\sum_{i=1}^{n} G_{i}\right|<\varepsilon .
$$

The statement that ${ }_{a} \Pi^{b}(1+G)$ exists means there exists an element $L$ of $N$ such that, if $\varepsilon>0$, then there exists a subdivision $D$ of $[a, b]$ such that, if $\left\{x_{i}\right\}_{i=0}^{n}$ is a refinement of $D$, then

$$
\left|L-\prod_{i=1}^{n}\left(1+G_{i}\right)\right|<\varepsilon .
$$

The statement that $(L R) \int_{a}^{b}(f G+f H)$ exists means $\int_{a}^{b} C$ exists, where $C(x, y)=f(x) G(x, y)+f(y) H(x, y)$ for $a<x<y<b$. The integral $(L R L R) \int_{a}^{b}(f H+G f+f K f)$ is defined similarly.

The statements that $G$ is bounded on $[a, b], G$ has bounded variation on $[a$, $b]$ and $G$ is in $O P^{\circ}$ on $[a, b]$ mean there exist a subdivision $D$ of $[a, b]$ and a number $B$ such that, if $\left\{x_{i}\right\}_{i=0}^{n}$ is a refinement of $D$, then

(1) $\left|G_{i}\right|<B$ for $i \neq 1,2, \ldots, n$,

(2) $\sum_{i=1}^{n}\left|G_{i}\right|<B$, and

(3) $\left|\Pi_{i=r+1}^{s}\left(1+G_{i}\right)\right|<B$ for $0<r<s<n$, respectively. 
The statement that $G$ is in $O M^{\circ}$ on $[a, b]$ means ${ }_{x} \Pi^{y}(1+G)$ exists for $a<x<y<b$ and $\int_{a}^{b}|1+G-\Pi(1+G)|$ exists and is zero. The statement that $G$ is in $O M^{*}$ on $[a, b]$ means ${ }_{x} \Pi^{y}(1+G)$ exists for $a \leqslant x<y<b$ and, if $\varepsilon>0$, then there exists a subdivision $D$ of $[a, b]$ such that, if $\left\{x_{i}\right\}_{i=0}^{n}$ is a refinement of $D$ and $0 \leqslant p<q \leqslant n$, then

$$
\left|x_{p} \Pi^{x_{q}}(1+G)-\prod_{i=p+1}^{q}\left(1+G_{i}\right)\right|<\varepsilon .
$$

The symbols $G\left(p^{-}, p\right), G\left(p^{-}, p^{-}\right), G\left(p, p^{+}\right)$and $G\left(p^{+}, p^{+}\right)$are used to denote

$$
\lim _{x \rightarrow p^{-}} G(x, p), \quad \lim _{x, y \rightarrow p^{-}} G(x, y), \quad \lim _{x \rightarrow p^{+}} G(p, x)
$$

and

$$
\lim _{x, y \rightarrow p^{+}} G(x, y),
$$

respectively. Further, the function $h$ is quasi-continuous on $[a, b]$ only if $\lim _{x \rightarrow p^{-}} h(x)$ exists for $a<p \leqslant b$ and $\lim _{x \rightarrow p^{+}} h(x)$ exists for $a \leqslant p<b$. For convenience in notation, let $G \in S_{1}$ on $[a, b]$ only if $G\left(p^{-}, p^{-}\right)$exists and is zero for $a<p \leqslant b$ and $G\left(p^{+}, p^{+}\right)$exists and is zero for $a \leqslant p<b$, and let $G \in S_{2}$ on $[a, b]$ only if $G\left(p^{-}, p\right)$ exists for $a<p \leqslant b$ and $G\left(p, p^{+}\right)$exists for $a<p<b$.

Results. The two existence theorems are presented first. One lemma is needed.

LEMma 1. If $S, T$ and $G$ are functions from $R \times R$ to $N$, each of $S\left(p^{-}, p\right)$, $S\left(p^{-}, p^{-}\right), T\left(p^{-}, p\right)$ and $T\left(p^{-}, p^{-}\right)$exists for $a<p \leqslant b$, each of $S\left(p, p^{+}\right)$, $S\left(p^{+}, p^{+}\right), T\left(p, p^{+}\right)$and $T\left(p^{+}, p^{+}\right)$exists for $a \leqslant p<b, G$ has bounded variation on $[a, b]$ and $\int_{x}^{y} G$ exists and is zero for $a \leqslant x<y \leqslant b$, then $\int_{a}^{b} S G T$ exists and is zero.

Proof. Let $\varepsilon>0$. It follows from the existence of the limits involving $S$ and $T$ by an application of the covering theorem that $S$ and $T$ are bounded on $[a, b]$. Thus, since $G$ has bounded variation on $[a, b]$, there exist a subdivision $D_{1}$ of $[a, b]$ and a number $B$ such that, if $\left\{x_{i}\right\}_{i=0}^{n}$ is a refinement of $D_{1}$, then

$$
\sum_{i=1}^{n}\left|G_{i}\right|\left|T_{i}\right|<B \text { and } \sum_{i=1}^{n}\left(\left|S_{i}\right|+1\right)\left|G_{i}\right|<B .
$$

Again, it follows from the existence of the limits involving $S$ and $T$ by an application of the covering theorem that there exist a subdivision $\left\{r_{j}\right\}_{j=0}^{p}$ of $[a, b]$ and sequences $\left\{s_{j}\right\}_{j=1}^{p}$ and $\left\{t_{j}\right\}_{j=1}^{p}$ of elements of $N$ such that, if $1 \leqslant j \leqslant p$ and $r_{j-1}<x<y<r_{j}$, then

$$
\left|S(x, y)-s_{j}\right|<\varepsilon(4 B)^{-1}, \quad\left|s_{j}\right|<|S(x, y)|+1
$$


and

$$
\left|T(x, y)-t_{j}\right|<\varepsilon(4 B)^{-1}
$$

Since $G$ has bounded variation on $[a, b], S$ and $T$ are bounded on $[a, b]$, $G\left(p, p^{+}\right)$exists and is zero for $a \leqslant p<b$ and $G\left(p^{-}, p\right)$ exists and is zero for $a<p \leqslant b$, it follows that there exist subdivisions $\left\{u_{j}\right\}_{j=0}^{p+1}$ and $\left\{v_{j}\right\}_{j=0}^{p+1}$ of $[a$, $b]$ such that $r_{j-1}<u_{j}<v_{j}<r_{j}$ for $j=1,2, \ldots, p$ and also such that

$$
\left|\sum_{j=1}^{p+1} \sum_{k=1}^{n(j)} S_{j k} G_{j k} T_{j k}\right|<\varepsilon / 4
$$

for refinements $\left\{x_{j k}\right\}_{k=0}^{n(j)}$ of $\left\{v_{j-1}, r_{j-1}, u_{j}\right\}$.

For $j=1,2, \ldots, p$, there exists a subdivision $E_{j}$ of $\left[u_{j}, v_{j}\right]$ such that, if $\left\{x_{i}\right\}_{i=0}^{n}$ is a refinement of $E_{j}$, then

$$
\left|\sum_{i=1}^{n} s_{j} G_{i} t_{j}\right|<\varepsilon(4 p)^{-1} .
$$

This is true since $\int_{u}^{v} s_{j} G t_{j}$ exists and is zero.

Let $D$ denote the subdivision

$$
D_{1} \cup D_{2} \bigcup_{j=1}^{p} E_{j} \cup\left\{r_{j}\right\}_{j=0}^{p}
$$

of $[a, b]$. Suppose $\left\{x_{i}\right\}_{i=0}^{n}$ is a refinement of $D$. For $j=1,2, \ldots, p$, let $P_{j}$ denote the set such that $i \in P_{j}$ only if $u_{j}<x_{i}<v_{j}$. Further, let $Q$ denote $\{i\}_{i=1}^{n}-\cup_{j=1}^{p} P_{j}$. Now,

$$
\begin{aligned}
\left|\sum_{i=1}^{n} S_{i} G_{i} T_{i}\right| \leqslant & \sum_{j=1}^{p}\left|\sum_{i \in P_{j}} S_{i} G_{i} T_{i}\right|+\left|\sum_{i \in Q} S_{i} G_{i} T_{i}\right| \\
& <\sum_{j=1}^{p}\left|\sum_{i \in P_{j}}\left(S_{i}-s_{j}+s_{j}\right) G_{i}\left(T_{i}-t_{j}+t_{j}\right)\right|+\varepsilon / 4 \\
& <\sum_{j=1}^{p}\left|\sum_{i \in P_{j}} s_{j} G_{i} t_{j}\right|+\sum_{j=1}^{p} \sum_{i \in P_{j}}\left|s_{j}\right|\left|G_{i}\right|\left|T_{i}-t_{j}\right| \\
& +\sum_{j=1}^{p} \sum_{i \in P_{j}}\left|S_{i}-s_{j}\right|\left|G_{i}\right|\left|T_{i}\right|+\varepsilon / 4 \\
& <p\left[\varepsilon(4 p)^{-1}\right]+\left[\varepsilon(4 B)^{-1}\right] B+\left[\varepsilon(4 B)^{-1}\right] B+\varepsilon / 4 \\
& =\varepsilon .
\end{aligned}
$$

Therefore, $\int_{a}^{b} S G T$ exists and is zero. This completes the proof of Lemma 1.

THEOREM 1. If $S, T$ and $G$ are functions from $R \times R$ to $N$, each of $S\left(p^{-}, p\right)$, $S\left(p^{-}, p^{-}\right), T\left(p^{-}, p\right)$ and $T\left(p^{-}, p^{-}\right)$exists for $a<p \leqslant b$, each of $S\left(p, p^{+}\right)$, 
$S\left(p^{+}, p^{+}\right), T\left(p, p^{+}\right)$and $T\left(p^{+}, p^{+}\right)$exists for $a<p<b, G$ has bounded variation on $[a, b]$ and $\int_{a}^{b} G$ exists, then

$$
\int_{a}^{b} S\left[G-\int G\right] T
$$

exists and is zero.

Proof. This theorem follows as a corollary to Lemma 1.

THEOREM 2. If $S, T$ and $G$ are functions from $R \times R$ to $N$, each of $S\left(p^{-}, p\right), s\left(p^{-}, p^{-}\right), T\left(p^{-}, p\right)$ and $T\left(p^{-}, p^{-}\right)$exists for $a<p<b$, each of $S\left(p, p^{+}\right), S\left(p^{+}, p^{+}\right), T\left(p, p^{+}\right)$and $T\left(p^{+}, p^{+}\right)$exists for $a<p<b, G$ has bounded variation on $[a, b]$ and ${ }_{x} \Pi^{y}(1+G)$ exists for $a<x<y<b$, then

$$
\int_{a}^{b} S[1+G-\Pi(1+G)] T
$$

exists and is zero.

Proof. This theorem follows as a corollary to Lemma 1.

The solution of the linear integral equation is now presented. Several additional lemmas are needed.

LEMMA 2. If $S, T$ and $G$ are functions from $R \times R$ to $N$, each of $S\left(p^{-}, p\right)$, $S\left(p^{-}, p^{-}\right), T\left(p^{-}, p\right)$ and $T\left(p^{-}, p^{-}\right)$exists for $a<p<b$, each of $S\left(p, p^{+}\right)$, $S\left(p^{+}, p^{+}\right), T\left(p, p^{+}\right)$and $T\left(p^{+}, p^{+}\right)$exists for $a<p<b, G$ has bounded variation on $[a, b]$ and $\int_{a}^{b} G$ exists, then $\int_{a}^{b} S G T$ exists [14, Theorem 5].

LEMMA 3. If $G$ is a function from $R \times R$ to $N$ and $G$ has bounded variation on $[a, b]$, then $\int_{a}^{b} G$ exists if and only if ${ }_{x} \Pi^{y}(1+G)$ exists for $a<x<y<b$ [14, Theorem 4].

LEMMA 4. If $h$ is a function from $R$ to $N, H$ and $L$ are functions from $R \times R$ to $N, h$ is quasi-continuous on $[a, b], H$ and $L-1$ have bounded variation on $[a, b], \int_{a}^{b} H$ exists and $\int_{a}^{b}(L-1)$ exists, then

$$
(R) \int_{a}^{b} d h L\left[\Pi_{0}^{b}(1+H)\right]
$$

exists.

Proof. For convenience in notation, let

$$
\begin{aligned}
& S_{1}(x, y)=(R) \int_{x}^{y} d h(L-1)\left[0 \Pi^{y}(1+H)\right] \\
& S_{2}(x, y)=\lim _{t \rightarrow x^{+}}(L R) \int_{t}^{y} h(u) H\left[0 \Pi^{y}(1+H)\right]
\end{aligned}
$$

and

$$
S_{3}(x, y)=\lim _{t \rightarrow x^{+}} h(x)\left[{ }_{t} \Pi^{y}(1+H)\right]
$$


for $a<x<y<b$. The existence of $S_{1}(x, y)$ and $S_{2}(x, y)$ can be established by using Lemma 2. For simplicity, $S_{1}, S_{2}$ and $S_{3}$ are used to denote $S_{1}(a, b)$, $S_{2}(a, b)$ and $S_{3}(a, b)$, respectively. In the following, it is shown that the desired integral exists and is

$$
S_{1}+S_{2}+h(b)-S_{3}
$$

Let $\varepsilon>0$.

If $\left\{x_{i}\right\}_{i=0}^{n}$ is a subdivision of $[a, b]$, then

$$
\begin{aligned}
& \sum_{i=1}^{n} d h_{i} L_{i}\left[x_{i} \Pi^{b}(1+H)\right] \\
&=\sum_{i=1}^{n} d h_{i}\left[L_{i}-1\right]\left[x_{i} \Pi^{b}(1+H)\right] \\
& \quad+\sum_{i=2}^{n} h_{i-1} H_{i}\left[x_{i} \Pi^{b}(1+H)\right]+h(b) \\
&-h(a)\left[x_{1} \Pi^{b}(1+H)\right]+\sum_{i=1}^{n-1} h_{i} e_{i}\left[x_{i+1} \Pi^{b}(1+H)\right]
\end{aligned}
$$

where

$$
e_{i}={ }_{x_{i}} \Pi^{x_{i+1}}(1+H)-\left(1+H_{i+1}\right)
$$

for $i=1,2, \ldots, n-1$. Now, by employing the preceding relation, we have that

$$
\left|\left\{\sum_{i=1}^{n} d h_{i} L_{i}\left[{ }_{x_{i}} \Pi^{b}(1+H)\right]\right\}-\left\{S_{1}+S_{2}+h(b)-S_{3}\right\}\right|
$$

$$
\begin{aligned}
& <\left|\sum_{i=1}^{n} d h_{i}\left[L_{i}-1\right]\left[x_{i} \Pi^{b}(1+H)\right]-S_{1}\right| \\
& +\left|\sum_{i=2}^{n} h_{i-1} H_{i}\left[x_{i} \Pi^{b}(1+H)\right]-S_{2}\right| \\
& +\left|S_{3}-h(a)\left[x_{1} \Pi^{b}(1+H)\right]\right| \\
& +\left|\sum_{i=1}^{n-1} h_{i} e_{i}\left[x_{i+1} \Pi^{b}(1+H)\right]\right|
\end{aligned}
$$

It follows from the existence of $S_{1}, S_{2}$ and $S_{3}$ that there exists a subdivision $D_{1}$ of $[a, b]$ such that, if $\left\{x_{i}\right\}_{i=0}^{n}$ is a refinement of $D_{1}$, then each of the expressions in (a), (b) and (c) is less than $\varepsilon / 4$. Further, it follows from Theorem 2 that there exists a subdivision $D_{2}$ of $[a, b]$ such that, if $\left\{x_{i}\right\}_{i=0}^{n}$ is a refinement of $D_{2}$, then the expression in (d) is less than $\varepsilon / 4$. Let $D$ denote the 
subdivision $D_{1} \cup D_{2}$ of $[a, b]$ and suppose $\left\{x_{i}\right\}_{i=0}^{n}$ is a refinement of $D$. Then,

$$
\begin{gathered}
\left|\left\{\sum_{i=1}^{n} d h_{i} L_{i}\left[{ }_{x_{i}} \Pi^{b}(1+H)\right]\right\}-\left\{S_{1}+S_{2}+h(b)-S_{3}\right\}\right| \\
<\varepsilon / 4+\varepsilon / 4+\varepsilon / 4+\varepsilon / 4=\varepsilon .
\end{gathered}
$$

Therefore, the desired integral exists and is

$$
S_{1}+S_{2}+h(b)-S_{3} \text {. }
$$

This completes the proof of Lemma 4.

LEMMA 5. If $G$ is a function from $R \times R$ to $N, G$ has bounded variation on $[a, b]$ and ${ }_{x} \Pi^{y}(1+G)$ exists for $a \leqslant x<y<b$, then $G$ is in $O M^{*}$ on $[a, b]$ [14, Theorem 1].

LEMMA 6. If $\varepsilon>0, h$ is a function from $R$ to $N, H$ and $L$ are functions from $R \times R$ to $N, h$ is quasi-continuous on $[a, b], H$ and $L-1$ have bounded variation on $[a, b], \int_{a}^{b} H$ exists and $\int_{a}^{b}(L-1)$ exists, then there exists a subdivision $D$ of $[a, b]$ such that, if $\left\{x_{i}\right\}_{i=0}^{n}$ is a refinement of $D$, then

$$
\left|\sum_{i=1}^{n} d h_{i} L_{i}\left[\prod_{j=i+1}^{n}\left(1+H_{j}\right)\right]-(R) \int_{a}^{b} d h L\left[{ }_{0} \Pi^{b}(1+H)\right]\right|<\varepsilon .
$$

Proof. It is established in Lemma 4 that

$$
\text { (R) } \int_{a}^{b} d h L\left[{ }_{0} \Pi^{b}(1+H)\right]=S_{1}+S_{2}+h(b)-S_{3} \text {, }
$$

where $S_{1}, S_{2}$ and $S_{3}$ are defined in Lemma 4. Also, if $\left\{x_{i}\right\}_{i=0}^{n}$ is a subdivision of $[a, b]$, then

$$
\begin{aligned}
\sum_{i=1}^{n} d h_{i} L_{i}\left[\prod_{j=i+1}^{n}\left(1+H_{j}\right)\right] \\
=\sum_{i=1}^{n} d h_{i}\left[L_{i}-1\right]\left[\prod_{j=i+1}^{n}\left(1+H_{j}\right)\right] \\
+\sum_{i=2}^{n} h_{i-1} H_{i}\left[\prod_{j=i+1}^{n}\left(1+H_{j}\right)\right]+h(b) \\
-h(a)\left[\prod_{j=2}^{n}\left(1+H_{j}\right)\right]
\end{aligned}
$$

Now, by employing the two preceding relations, we have that 
(a)

$$
\left|\sum_{i=1}^{n} d h_{i} L_{i}\left[\prod_{j=i+1}^{n}\left(1+H_{j}\right)\right]-(R) \int_{a}^{b} d h L\left[{ }_{0} \Pi^{b}(1+H)\right]\right|
$$

$$
\leqslant\left|\sum_{i=1}^{n} d h_{i}\left[L_{i}-1\right]\left[\prod_{j=i+1}^{n}\left(1+H_{j}\right)\right]-S_{1}\right|
$$

(b)

$$
+\left|\sum_{i=2}^{n} h_{i-1} H_{i}\left[\prod_{j=i+1}^{n}\left(1+H_{j}\right)\right]-S_{2}\right|
$$

(c)

$$
+\left|S_{3}-h(a)\left[\prod_{j=2}^{n}\left(1+H_{j}\right)\right]\right| \text {. }
$$

Let $\varepsilon>0$. Since $L-1$ has bounded variation on $[a, b]$ and $H$ is in $O M^{*}$ on $[a, b]$ by Lemma 5 , there exists a subdivision $D_{1}$ of $[a, b]$ such that, if $\left\{x_{i}\right\}_{i=0}^{n}$ is a refinement of $D_{1}$, then the expression in (a) is less than $\varepsilon / 3$. Similarly, since $H$ has bounded variation on $[a, b]$, there exists a subdivision $D_{2}$ of $[a, b]$ such that, if $\left\{x_{i}\right\}_{i=0}^{n}$ is a refinement of $D_{2}$, then the expression in (b) is less than $\varepsilon / 3$. Finally, from the existence of $S_{3}$, it follows that there exists a subdivision $D_{3}$ of $[a, b]$ such that, if $\left\{x_{i}\right\}_{i=0}^{n}$ is a refinement of $D_{3}$, then the expression in (c) is less than $\varepsilon / 3$.

Let $D$ denote the subdivision $D_{1} \cup D_{2} \cup D_{3}$ of $[a, b]$ and suppose $\left\{x_{i}\right\}_{i=0}^{n}$ is a refinement of $D$. Then,

$$
\begin{gathered}
\left|\sum_{i=1}^{n} d h_{i} L_{i}\left[\prod_{j=i+1}^{n}\left(1+H_{j}\right)\right]-(R) \int_{a}^{b} d h L\left[{ }_{0} \Pi^{b}(1+H)\right]\right| \\
<\varepsilon / 3+\varepsilon / 3+\varepsilon / 3=\varepsilon .
\end{gathered}
$$

Therefore, the desired inequality is established. This completes the proof of Lemma 6.

LEMMA 7. If $h$ is a function from $R$ to $N, H$ and $L$ are functions from $R \times R$ to $N, h$ is quasi-continuous on $[a, b], H$ and $L-1$ have bounded variation on $[a, b], \int_{a}^{b} H$ exists and $\int_{a}^{b}(L-1)$ exists, then $\int_{a}^{b}|d h L-I|$ exists and is zero, where

$$
I(x, y)=(R) \int_{x}^{y} d h L\left[0 \Pi^{y}(1+H)\right]
$$

for $a \leqslant x<y \leqslant b$.

Proof. It is established in Lemma 4 that

$$
I(x, y)=S_{1}(x, y)+S_{2}(x, y)+h(y)-S_{3}(x, y)
$$

for $a \leqslant x<y \leqslant b$, where $S_{1}(x, y), S_{2}(x, y)$ and $S_{3}(x, y)$ are defined in Lemma 4. Thus, if $\left\{x_{i}\right\}_{i=0}^{n}$ is a subdivision of $[a, b]$, then 


$$
\sum_{i=1}^{n}\left|d h_{i} L_{i}-I\left(x_{i-1}, x_{i}\right)\right|
$$

(a)

$$
<\sum_{i=1}^{n}\left|d h_{i}\left(L_{i}-1\right)-S_{1}\left(x_{i-1}, x_{i}\right)\right|
$$

$$
+\sum_{i=1}^{n}\left|S_{3}\left(x_{i-1}, x_{i}\right)-h_{i-1}-S_{2}\left(x_{i-1}, x_{i}\right)\right| \text {. }
$$

We now establish that the desired integral exists and is zero. Let $\varepsilon>0$.

For any specified positive number $\alpha$, there exists a subdivision $\left\{x_{i}\right\}_{i=0}^{n}$ of $[a, b]$ such that, if $x_{i-1}<u_{i}<v_{i}<x_{i}$ and $\left\{x_{i j}\right\}_{j=0}^{n(i)}$ is a subdivision of $\left[u_{i}, v_{i}\right]$ for $i=1,2, \ldots, n$, then

$$
\sum_{i=1}^{n} \sum_{j=1}^{n(i)}\left|d h_{i j}\left(L_{i j}-1\right)\right|<\alpha .
$$

By using this as a starting point, it is possible to construct a subdivision $D_{1}$ of $[a, b]$ such that, if $\left\{x_{i}\right\}_{i=0}^{n}$ is a refinement of $D_{1}$, then the expression in (a) is less than $\varepsilon / 2$.

We next note two things. First,

$$
{ }_{x} \Pi^{y}(1+H)=1+(R) \int_{x}^{y} H\left[{ }_{0} \Pi^{y}(1+H)\right]
$$

for $a<x<y<b$. Second, if $\alpha$ is a positive number, then there exists a subdivision $\left\{x_{i}\right\}_{i=0}^{n}$ of $[a, b]$ such that, if $x_{i-1}<u_{i}<v_{i}<x_{i}$ for $i=1$, $2, \ldots, n$, then

$$
\sum_{i=1}^{n}\left|(L R) \int_{u_{i}}^{v_{i}}\left[h\left(u_{i}\right)-h(u)\right] H\left[{ }_{v} \Pi^{v_{i}}(1+H)\right]\right|<\alpha .
$$

By employing the two preceding relations, it is possible to construct a subdivision $D_{2}$ of $[a, b]$ such that, if $\left\{x_{i}\right\}_{i=0}^{n}$ is a refinement of $D_{2}$, then the expression in (b) is less than $\varepsilon / 2$.

Let $D$ denote the subdivision $D_{1} \cup D_{2}$ of $[a, b]$ and suppose $\left\{x_{i}\right\}_{i=0}^{n}$ is a refinement of $D$. Then,

$$
\sum_{i=1}^{n}\left|d h_{i} L_{i}-I\left(x_{i-1}, x_{i}\right)\right|<\varepsilon / 2+\varepsilon / 2=\varepsilon .
$$

Therefore, the desired integral exists and is zero. This completes the proof of Lemma 7.

THEOREM 3. If $f$ and $h$ are functions from $R$ to $N, G, H, A$ and $B$ are functions from $R \times R$ to $N,(1-H)^{-1}$ exists and is bounded on $[a, b]$, $B=(1-H)^{-1}$ and $A=(1+G)(1-H)^{-1}$ on $[a, b], h$ is quasi-continuous on $[a, b], G$ and $H$ have bounded variation on $[a, b], \int_{a}^{b} G$ exists and $\int_{a}^{b} H$ exists, then the following statements are equivalent: 
(1) $f$ is bounded on $[a, b]$ and $(L R) \int_{a}^{x}(f G+f H)$ exists and is $f(x)-h(x)$ for $a<x<b$, and

(2) $(R) \int_{a}^{x}\left(d h B_{v} \Pi^{x} A\right)$ exists and is $f(x)-h(a)_{a} \Pi^{x} A$ for $a \leqslant x \leqslant b$.

Proof [(1) $\rightarrow$ (2)]. It follows that ${ }_{x} \Pi^{y} A$ exists for $a<x<y<b$ by employing Lemmas 2 and 3. Further, the existence of $(R) \int_{a}^{x}\left(d h B_{v} \Pi^{x} A\right)$ for $a<x<b$ follows from Lemma 4. We now establish that

$$
f(x)=h(a)_{a} \Pi^{x} A+(R) \int_{a}^{x}\left(d h B_{v} \Pi^{x} A\right)
$$

for $a<x<b$. If $a=x$, the equality follows immediately.

Suppose $a<x \leqslant b$ and $\varepsilon>0$. If $D$ is a subdivision of $[a, x]$ and $\left\{x_{i}\right\}_{i=0}^{n}$ is a refinement of $D$, then by employing an iteration technique used by B. W. Helton [7, Theorem 5.1, p. 311], we have that

$$
f_{n}=f_{0} \prod_{i=1}^{n} A_{i}+\sum_{i=1}^{n} d h_{i} B_{i} \prod_{j=i+1}^{n} A_{j}+\sum_{i=1}^{n} c_{i} B_{i} \prod_{j=i+1}^{n} A_{j}
$$

where

$$
c_{i}=(L R) \int_{x_{i-1}}^{x_{i}}(f G+f H)-\left(f_{i-1} G_{i}+f_{i} H_{i}\right)
$$

for $i=1,2, \ldots, n$. Thus,

(a)

$$
\begin{aligned}
\left|f(x)-\left[h(a)_{a} \Pi^{x} A+(R) \int_{a}^{x}\left(d h B_{v} \Pi^{x} A\right)\right]\right| \\
\leqslant\left|h(a) \prod_{i=1}^{n} A_{i}-h(a)_{a} \Pi^{x} A\right| \\
\quad+\left|\sum_{i=1}^{n} d h_{i} B_{i} \prod_{j=i+1}^{n} A_{j}-(R) \int_{a}^{x}\left(d h B_{v} \Pi^{x} A\right)\right| \\
\quad+\left|\sum_{i=1}^{n} c_{i} B_{i} \prod_{j=i+1}^{n} A_{j}\right| .
\end{aligned}
$$

Since ${ }_{a} \Pi^{x} A$ exists, there exists a subdivision $D_{1}$ of $[a, x]$ such that, if $\left\{x_{i}\right\}_{i=0}^{n}$ is a refinement of $D_{1}$, then the expression in (a) is less than $\varepsilon / 3$. Further, it follows from Lemma 6 that there exists a subdivision $D_{2}$ of $[a, x]$ such that, if $\left\{x_{i}\right\}_{i=0}^{n}$ is a refinement of $D_{2}$, then the expression in (b) is less than $\varepsilon / 3$. It follows from Lemma 5 that $A-1$ is in $O M^{*}$ on $[a, x]$. Now, from this and Theorem 1, it follows that there exists a subdivision $D_{3}$ of $[a, x]$ such that, if $\left\{x_{i}\right\}_{i=0}^{n}$ is a refinement of $D_{3}$, then the expression in (c) is less than $\varepsilon / 3$.

Let $D$ denote the subdivision $\cup_{i=1}^{3} D_{i}$ of $[a, x]$. Thus, if $\left\{x_{i}\right\}_{i=0}^{n}$ is a refinement of $D$, then 


$$
\begin{aligned}
& \mid f(x)- {\left[h(a)_{a} \Pi^{x} A+(R) \int_{a}^{x}\left(d h B_{v} \Pi^{x} A\right)\right] \mid } \\
&<\varepsilon / 3+\varepsilon / 3+\varepsilon / 3=\varepsilon .
\end{aligned}
$$

Therefore, (1) implies (2).

Proof [(2) $\rightarrow(1)]$. It follows from the definition of $f$ in (2) that $f$ is bounded on $[a, b]$. The existence of $(L R) \int_{a}^{x}(f G+f H)$ for $a \leqslant x \leqslant b$ follows from Lemma 2. We now establish that

$$
f(x)=h(x)+(L R) \int_{a}^{x}(f G+f H)
$$

for $a \leqslant x \leqslant b$. If $a=x$, the equality follows immediately.

Suppose $a<x \leqslant b$ and $\varepsilon>0$. If $D$ is a subdivision of $[a, x]$ and $\left\{x_{i}\right\}_{i=0}^{n}$ is a refinement of $D$, then by employing an iteration technique used by B. W. Helton [7, Theorem 5.1, p. 312], we have that

$$
f_{n}=h_{n}+\sum_{i=1}^{n}\left(f_{i-1} G_{i}+f_{i} H_{i}\right)+\sum_{i=1}^{n} f_{i-1} d_{i}\left(1-H_{i}\right)+\sum_{i=1}^{n} e_{i}\left(1-H_{i}\right),
$$

where

$$
d_{i}={ }_{x_{i-1}} \Pi^{x_{i}} A-A_{i}
$$

and

$$
e_{i}=(R) \int_{x_{i-1}}^{x_{i}}\left(d h B_{v} \Pi^{x_{i}} A\right)-d h_{i} B_{i}
$$

for $i=1,2, \ldots, n$. Thus,

$$
\begin{aligned}
\left|f(x)-\left[h(x)+(L R) \int_{a}^{x}(f G+f H)\right]\right| \\
\leqslant\left|\sum_{i=1}^{n}\left(f_{i-1} G_{i}+f_{i} H_{i}\right)-(L R) \int_{a}^{x}(f G+f H)\right| \\
\quad+\left|\sum_{i=1}^{n} f_{i-1} d_{i}\left(1-H_{i}\right)\right| \\
\quad+\sum_{i=1}^{n}\left|e_{i}\left(1-H_{i}\right)\right| .
\end{aligned}
$$

Since $(L R) \int_{a}^{x}(f G+f H)$ exists, there exists a subdivision $D_{1}$ of $[a, x]$ such that, if $\left\{x_{i}\right\}_{i=0}^{n}$ is a refinement of $D_{1}$, then the expression in (a) is less than $\varepsilon / 3$. Further, it follows from Theorem 2 that there exists a subdivision $D_{2}$ of $[a, x]$ such that, if $\left\{x_{i}\right\}_{i=0}^{n}$ is a refinement of $D_{2}$, then the expression in (b) is less than $\varepsilon / 3$. Finally, it follows from Lemma 7 that there exists a subdivision $D_{3}$ of $[a, x]$ such that, if $\left\{x_{i}\right\}_{i=0}^{n}$ is a refinement of $D_{3}$, then the expression in (c) is less than $\varepsilon / 3$. 
Let $D$ denote the subdivision $\cup_{i=1}^{3} D_{i}$ of $[a, x]$. Thus, if $\left\{x_{i}\right\}_{i=0}^{n}$ is a refinement of $D$, then

$$
\left|f(x)-\left[h(x)+(L R) \int_{a}^{x}(f G+f H)\right]\right|<\varepsilon / 3+\varepsilon / 3+\varepsilon / 3=\varepsilon .
$$

Therefore, (2) implies (1). This completes the proof of Theorem 3.

The solution of the Riccati integral equation is now presented. Several additional lemmas are needed.

LEMMA 8. If $F$ and $G$ are functions from $R \times R$ to $N, F$ is in $O P^{\circ}, O M^{*}$ and $S_{1} \cap S_{2}$ on $[a, b], G$ has bounded variation on $[a, b]$ and $G$ is in $O M^{*}$ on $[a, b]$, then $F+G$ is in $O P^{\circ}$ and $O M^{*}$ on $[a, b][17$, Lemma 5, Theorem 1].

LEMMA 9. If $F$ and $G$ are functions from $R \times R$ to $N, F$ is in $O P^{\circ}, O M^{*}$ and $S_{1} \cap S_{2}$ on $[a, b], G$ has bounded variation on $[a, b]$ and $G$ is in $O M^{*}$ on $[a, b]$, then

$$
(L R) \int_{a}^{b}\left[{ }_{a} \Pi^{r}(1+F)\right] G(r, s)\left[\Pi^{b}(1+F+G)\right]
$$

exists and is

$$
{ }_{a} \Pi^{b}(1+F+G)-{ }_{a} \Pi^{b}(1+F) .
$$

Proof. It follows from Lemma 8 that $F+G$ is in $O P^{\circ}$ and $O M^{*}$ on $[a, b]$. Further, it can be established by induction that, if $\left\{x_{i}\right\}_{i=0}^{n}$ is a subdivision of $[a, b]$, then

$$
\begin{array}{r}
\sum_{i=1}^{n}\left[\prod_{j=1}^{i-1}\left(1+F_{j}\right)\right] G_{i}\left[\prod_{j=i+1}^{n}\left(1+F_{j}+G_{j}\right)\right] \\
=\prod_{i=1}^{n}\left(1+F_{i}+G_{i}\right)-\prod_{i=1}^{n}\left(1+F_{i}\right) .
\end{array}
$$

The existence of the desired integral can be established by using the preceding identity and the facts that $G$ has bounded variation on $[a, b]$ and that $F$ and $F+G$ are in $O P^{\circ}$ and $O M^{*}$ on $[a, b]$. This completes the proof of Lemma 9.

LEMMA 10. If $G$ is a function from $R \times R$ to $N, G$ has bounded variation on $[a, b],{ }_{x} \Pi^{y}(1+G)$ exists for $a \leqslant x<y \leqslant b$ and $(1+G)^{-1}$ exists and is bounded on $[a, b]$, then $\left[{ }_{x} \Pi^{y}(1+G)\right]^{-1}$ exists and is bounded on $[a, b]$.

Proof. If $A$ is an element of $N$ and $|A|<1$, then

$$
(1+A)^{-1}=1+\sum_{j=1}^{\infty}(-1)^{j} A^{j}
$$

By employing this relation, we can establish that, if $G$ satisfies the conditions 
in the hypothesis on some interval $[u, v]$ plus the additional condition that $|G|<\frac{1}{2}$ on that interval, then $\left[\Pi_{u} \Pi^{v}(1+G)\right]^{-1}$ exists and is ${ }_{v}^{u}(1+H)$, where

$$
H(s, r)=\sum_{j=1}^{\infty}(-1)^{j} G^{j}(r, s)
$$

for $u<r<s \leqslant v$.

Since $G$ has bounded variation on $[a, b]$, there exists a subdivision $\left\{x_{i}\right\}_{i=0}^{n}$ of $[a, b]$ such that, if $1<i<n$ and $x_{i-1}<u<v<x_{i}$, then $|G|<\frac{1}{2}$ on $[u$, v]. Hence, the existence of $\left[\Pi_{x} \Pi^{y}(1+G)\right]^{-1}$ for $a<x_{0}<y<b$ can be established from the existence of $(1+G)^{-1}$ and the result given in the previous paragraph. Further, since $G$ has bounded variation, it also follows that this inverse is bounded on $[a, b]$. This completes the proof of Lemma 10.

LEMMA 11. If $F, G$ and $L$ are functions from $R \times R$ to $N, F$ is in $O P^{\circ}, O M^{\circ}$ and $S_{1} \cap S_{2}$ on $[a, b], G$ has bounded variation on $[a, b], \int_{a}^{b} G$ exists and

$$
L(r, s)={ }_{r} \Pi^{s}(1+F+G)-1-F(r, s)-G(r, s)
$$

for $a<r<s<b$, then $L$ has bounded variation on $[a, b]$ and $\int_{x}^{y} L$ exists and is zero for $a \leqslant x<y \leqslant b$.

Proof. It is initially established that $\int_{x}^{y} L$ exists and is zero for $a<x<y$ $<b$. Suppose $a<x<y<b$ and $\varepsilon>0$. By applying Lemma 9, we have that, if $\left\{x_{i}\right\}_{i=0}^{n}$ is a subdivision of $[x, y]$, then

$\left|\sum_{i=1}^{n} L_{i}\right|=\left|\sum_{i=1}^{n} x_{i-1} \Pi^{x_{i}}(1+F+G)-\left(1+F_{i}+G_{i}\right)\right|$

$$
<\left|\sum_{i=1}^{n} x_{i-1} \Pi^{x_{i}}(1+F)-\left(1+F_{i}\right)\right|
$$

(b) $+\left|\sum_{i=1}^{n}(L R) \int_{x_{i-1}}^{x_{i}}\left[x_{i-1} \Pi^{r}(1+F)\right] G(r, s)\left[s \Pi^{x_{i}}(1+F+G)\right]-G\right|$.

Since $F$ is in $O M^{\circ}$ on $[x, y]$, there exists a subdivision $D_{1}$ of $[x, y]$ such that, if $\left\{x_{i}\right\}_{i=0}^{n}$ is a refinement of $D_{1}$, then the expression in (a) is less than $\varepsilon / 2$.

For any specified positive number $\alpha$, there exists a subdivision $\left\{x_{i}\right\}_{i=0}^{n}$ of $[x, y]$ such that, if $1<i<n$ and $x_{i-1}<u<v<x_{i}$, then

$$
\left|1-{ }_{u} \Pi^{0}(1+F)\right|<\alpha \text { and }\left|1-{ }_{u} \Pi^{0}(1+F+G)\right|<\alpha .
$$

Hence, it is possible to construct a subdivision $D_{2}$ of $[x, y]$ such that, if $\left\{x_{i}\right\}_{i=0}^{n}$ is a refinement of $D_{2}$, then the expression in (b) is less than $\varepsilon / 2$.

Let $D$ denote the subdivision $D_{1} \cup D_{2}$ of $[x, y]$ and suppose $\left\{x_{i}\right\}_{i=0}^{n}$ is a refinement of $D$. Then, 


$$
\left|\sum_{i=1}^{n} L_{i}\right|<\varepsilon / 2+\varepsilon / 2=\varepsilon .
$$

Hence, $\int_{x}^{y} L$ exists and is zero.

It is now established that $L$ has bounded variation on $[a, b]$. Again by applying Lemma 9 , we have that, if $\left\{x_{i}\right\}_{i=0}^{n}$ is a subdivision of $[a, b]$, then

$$
\begin{aligned}
& \sum_{i=1}^{n}\left|L_{i}\right|=\sum_{i=1}^{n}\left|x_{i-1} \Pi^{x_{i}}(1+F+G)-\left(1+F_{i}+G_{i}\right)\right| \\
& <\sum_{i=1}^{n}\left|x_{i-1} \Pi^{x_{i}}(1+F)-\left(1+F_{i}\right)\right| \\
& \text { (a) } \quad+\sum_{i=1}^{n}\left|(L R) \int_{x_{i-1}}^{x_{i}}\left[x_{i-1} \Pi^{r}(1+F)\right] G(r, s)\left[{ }_{s} \Pi^{x_{i}}(1+F+G)\right]\right| \\
& \text { (b) } \quad+\sum_{i=1}^{n}\left|-G_{i}\right| \text {. }
\end{aligned}
$$

Since $F$ is in $O M^{\circ}$ on $[a, b]$, there exist a subdivision $D_{1}$ of $[a, b]$ and a number $B_{1}$ such that, if $\left\{x_{i}\right\}_{i=0}^{n}$ is a refinement of $D_{1}$, then the expression in (a) is less than $B_{1}$. Since $G$ has bounded variation on $[a, b]$ and each of $F$ and $F+G$ is in $O P^{\circ}$ on $[a, b]$, there exist a subdivision $D_{2}$ of $[a, b]$ and numbers $B_{2}$ and $B_{3}$ such that, if $\left\{x_{i}\right\}_{i=0}^{n}$ is a refinement of $D_{2}$, then the expressions in (b) and (c) are less than $B_{2}$ and $B_{3}$, respectively.

Let $D$ denote the subdivision $D_{1} \cup D_{2}$ of $[a, b]$ and suppose $\left\{x_{i}\right\}_{i=0}^{n}$ is a refinement of $D$. Then,

$$
\sum_{i=1}^{n}\left|L_{i}\right|<B_{1}+B_{2}+B_{3} .
$$

Hence, $L$ has bounded variation on $[a, b]$. This completes the proof of Lemma 11.

THEOREM 4. If $w$ is a function from $R$ to $N$, each of $G, H$ and $K$ is a function from $R \times R$ to $N, w$ is quasi-continuous on $[a, b]$, each of $G, H$ and $K$ has bounded variation on $[a, b], K\left(p, p^{+}\right)$exists and is zero for $a<p<b$, $K\left(p^{-}, p\right)$ exists and is zero for $a<p<b,(1-G)^{-1}$ exists and is bounded on $[a, b]$ and each of $\int_{a}^{b} G, \int_{a}^{b} H$ and $\int_{a}^{b} K$ exists, then the following statements are equivalent:

(1) the integral equation

$$
f(x)=w(x)+(L R L R) \int_{a}^{x}(f H+G f+f K f)
$$

has a bounded solution on $[a, b]$, and

(2) the inverse of $v(x)$ exists and is bounded on $[a, b]$, where $u(x)$ and $v(x)$ are defined by the equation 


$$
[u(x), v(x)]=[w(a), 1]_{a} \Pi^{x}\left(\mathbf{I}+\left[\begin{array}{cc}
H & -K \\
d w & -G
\end{array}\right]\right)
$$

for $a \leqslant x \leqslant b$.

Furthermore, if (1) or (2) is true, then the only bounded solution of the integral equation in (1) is given by $f(x)=[v(x)]^{-1} u(x)$ for $a<x \leqslant b$.

Proof. To simplify notation, let $\mathbf{Q}$ denote the function such that

$$
\mathbf{Q}(x, y)=\mathbf{I}+\left[\begin{array}{cc}
H(x, y) & -K(x, y) \\
w(y)-w(x) & -G(x, y)
\end{array}\right]
$$

for $a \leqslant x<y \leqslant b$. Then, if we let

$$
\mathbf{F}(x, y)=\left[\begin{array}{cc}
0 & 0 \\
w(y)-w(x) & 0
\end{array}\right] \text { and } \mathbf{G}(x, y)=\left[\begin{array}{cc}
H(x, y) & -K(x, y) \\
0 & -G(x, y)
\end{array}\right]
$$

for $a \leqslant x<y \leqslant b$, it follows from Lemmas 5 and 8 that $\mathbf{Q}$ is in $O P^{\circ}$ and $O M^{*}$ on $[a, b]$.

Proof [(1) $\rightarrow(2)]$. Suppose $a \leqslant x \leqslant b$. If $a=x$, then the result follows immediately. Therefore, suppose $a<x$. The existence of $(L) \int_{a}^{x}-f K$ follows from Lemma 2. Thus, $(L) \int_{a}^{x}(-G-f K)$ exists. Therefore, the existence of $(L)_{a} \Pi^{x}(1-G-f K)$ follows from Lemma 3 . We now establish that

$$
\left\{(L)_{a} \Pi^{x}(1-G-f K)\right\}[f(x), 1]=[u(x), v(x)]
$$

Let $\varepsilon>0$. If $L$ denotes the interval function

$$
\begin{aligned}
L(r, s)= & (L R L R) \int_{r}^{s}[f H+G f+f K f] \\
& -[f(r) H(r, s)+G(r, s) f(s)+f(r) K(r, s) f(s)]
\end{aligned}
$$

$D$ is a subdivision of $[a, x]$ and $\left\{x_{i}\right\}_{i=0}^{n}$ is a refinement of $D$ such that $\left(1-G_{i}-f_{i-1} K_{i}\right)^{-1}$ exists for $i=1,2, \ldots, n$, then by employing an iteration technique used by B. W. Helton [11, Theorem 3.3], we have that

$$
\begin{array}{r}
\left\{(L)_{a} \Pi^{x}(1-G-f K)+d_{1}\right\}\left[f_{n}, 1\right] \\
=\left[f_{0}, 1\right]\left\{{ }_{a} \Pi^{x} \mathbf{Q}+\mathbf{d}_{2}+\mathbf{d}_{3}\right\},
\end{array}
$$


where

$$
\begin{gathered}
d_{1}=\prod_{i=1}^{n}\left(1-G_{i}-f_{i-1} K_{i}\right)-(L)_{a} \Pi^{x}(1-G-f K) \\
\mathbf{d}_{2}=\prod_{i=1}^{n} \mathbf{Q}_{i}-{ }_{a} \prod^{x} \mathbf{Q}
\end{gathered}
$$

and

$$
\mathbf{d}_{3}=\prod_{i=1}^{n}\left(\mathrm{I}+\left[\begin{array}{cc}
H_{i} & -K_{i} \\
\Delta w_{i} & -G_{i}
\end{array}\right]+\left[\begin{array}{ll}
0 & 0 \\
L_{i} & 0
\end{array}\right]\right)-\prod_{i=1}^{n} \mathbf{Q}_{i} \text {. }
$$

Thus,

$$
\begin{aligned}
& \|\left\{(L)_{a} \Pi^{x}\right.(1-G-f K)\}\left[f_{n}, 1\right]-\left[f_{0}, 1\right]_{a} \Pi^{x} \mathbf{Q} \| \\
&=\left\|-d_{1}\left[f_{n}, 1\right]+\left[f_{0}, 1\right]\left\{\mathbf{d}_{2}+\mathbf{d}_{3}\right\}\right\| \\
&<\left|-d_{1}\right|\left\|\left[f_{n}, 1\right]\right\| \\
& \quad+\left\|\mathbf{d}_{2}\right\|\left\|\left[f_{0}, 1\right]\right\| \\
& \quad+\left\|\mathbf{d}_{3}\right\|\left\|\left[f_{0}, 1\right]\right\| .
\end{aligned}
$$

Since $(1-G)^{-1}$ exists on $[a, b], f$ is bounded on $[a, b]$ and each of $K\left(p, p^{+}\right)$and $K\left(p^{-}, p\right)$ exists and is zero on $[a, b]$, it follows that there exists a subdivision $D_{1}$ of $[a, x]$ such that, if $\left\{x_{i}\right\}_{i=0}^{n}$ is a refinement of $D_{1}$, then $\left(1-G_{i}-f_{i-1} K_{i}\right)^{-1}$ exists for $i=1,2, \ldots, n$. It follows from the existence of $(L)_{a} \Pi^{x}(1-G-f K)$ that there exists a subdivision $D_{2}$ of $[a, x]$ such that, if $\left\{x_{i}\right\}_{i=0}^{n}$ is a refinement of $D_{2}$, then the expression in (a) is less than $\varepsilon / 3$. It follows from the existence of ${ }_{a} \Pi^{x} Q$ that there exists a subdivision $D_{3}$ of $[a, x]$ such that, if $\left\{x_{i}\right\}_{i=0}^{n}$ is a refinement of $D_{3}$, then the expression in (b) is less than $\varepsilon / 3$. It follows from Lemma 9 and Theorem 1 that there exists a subdivision $D_{4}$ of $[a, x]$ such that, if $\left\{x_{i}\right\}_{i=0}^{n}$ is a refinement of $D_{4}$, then the expression in (c) is less than $\varepsilon / 3$.

Let $D$ denote the subdivision $\cup_{i=1}^{4} D_{i}$ of $[a, x]$. Thus, if $\left\{x_{i}\right\}_{i=0}^{n}$ is a refinement of $D$, then

$$
\begin{gathered}
\left\|\left\{(L)_{a} \Pi^{x}(1-G-f K)\right\}\left[f_{n}, 1\right]-\left[f_{0}, 1\right]_{a} \Pi^{x} \mathbf{Q}\right\| \\
<\varepsilon / 3+\varepsilon / 3+\varepsilon / 3=\varepsilon .
\end{gathered}
$$

Hence,

$$
\left\{(L)_{a} \Pi^{x}(1-G-f K)\right\}[f(x), 1]=[w(a), 1]_{a} \Pi^{x} \mathbf{Q}=[u(x), v(x)] .
$$


From the definition of matrix equality, we have that

$$
\left\{(L)_{a} \Pi^{x}(1-G-f K)\right\} f(x)=u(x)
$$

and

$$
(L)_{a} \Pi^{x}(1-G-f K)=v(x) .
$$

From Lemma 10 , we have that $\left\{(L)_{a} \Pi^{x}(1-G-f K)\right\}^{-1}$ exists. Thus, $[v(x)]^{-1}$ exists, and hence, $f(x)=[v(x)]^{-1} u(x)$. Also, note that Lemma 10 can be used to establish that $v^{-1}$ is bounded on $[a, b]$. Therefore, (1) implies (2). Also, we have shown that, if $f$ is a bounded solution to the integral equation in (1), then $f(x)=[v(x)]^{-1} u(x)$ for $a<x<b$.

Proof [(2) $\rightarrow(1)]$. Let $f(x)=[v(x)]^{-1} u(x)$ for $a<x<b$. Since $v^{-1}$ and $u$ are bounded on $[a, b]$, the funcion $f$ is bounded on $[a, b]$. Suppose $a<x<$ $b$. If $a=x$, then (1) follows immediately. Therefore, suppose $a<x$. We now establish that the equality in (1) is satisfied. Let $\varepsilon>0$.

If $D$ is a subdivision of $[a, x]$ and $\left\{x_{i}\right\}_{i=0}^{n}$ is a refinement $D$, then by employing an iteration technique used by B. W. Helton [11, Theorem 3.2], we have that

$$
\begin{aligned}
f_{n}= & w_{n}+\sum_{i=1}^{n} f_{i-1} H_{i}+\sum_{i=1}^{n} G_{i} f_{i}+\sum_{i=1}^{n} f_{i-1} K_{i} f_{i} \\
& +\sum_{i=1}^{n}\left(v_{i-1}^{-1} a_{i}-v_{i-1}^{-1} b_{i} f_{i}\right),
\end{aligned}
$$

where

$$
\left[a_{i}, b_{i}\right]=\left[u_{i-1}, v_{i-1}\right]\left\{x_{i-1} \Pi^{x_{i}} \mathbf{Q}-\mathbf{Q}_{i}\right\}
$$

for $i=1,2, \ldots, n$. To simplify notation, let $\mathrm{L}, S, T, U$ and $V$ denote the functions such that, if $a<r<s<x$, then

$$
\mathbf{L}(r, s)=\left[\begin{array}{ll}
S(r, s) & T(r, s) \\
U(r, s) & V(r, s)
\end{array}\right]={ }_{r} \Pi^{s} \mathbf{Q}-\mathbf{Q}(r, s) .
$$

Now, from the definitions of the functions involved, we have that

$$
v_{i-1}^{-1} a_{i}=v_{i-1}^{-1} u_{i-1} S_{i}+U_{i}
$$

and

$$
-v_{i-1}^{-1} b_{i} f_{i}=-v_{i-1}^{-1} u_{i-1} T_{i} v_{i}^{-1} u_{i}-V_{i} v_{i}^{-1} u_{i}
$$

Thus, 


$$
\begin{aligned}
\mid f(x)- & \left\{w(x)+\sum_{i=1}^{n}\left(f_{i-1} H_{i}+G_{i} f_{i}+f_{i-1} K_{i} f_{i}\right)\right\} \mid \\
& =\left|\sum_{i=1}^{n}\left(v_{i-1}^{-1} a_{i}-v_{i-1}^{-1} b_{i} f_{i}\right)\right|
\end{aligned}
$$

(a)

$$
\begin{gathered}
<\left|\sum_{i=1}^{n} v_{i-1}^{-1} u_{i-1} S_{i}\right| \\
+\left|\sum_{i=1}^{n} U_{i}\right|
\end{gathered}
$$

(b)

(c)

$$
\begin{aligned}
& +\left|\sum_{i=1}^{n}-v_{i-1}^{-1} u_{i-1} T_{i} v_{i}^{-1} u_{i}\right| \\
& +\left|\sum_{i=1}^{n}-V_{i} v_{i}^{-1} u_{i}\right| .
\end{aligned}
$$

It follows from Lemma 11 that $L$ has bounded variation on $[a, x]$ and that $\int_{r}^{s} \mathrm{~L}=0$ for $a<r<s<x$. Thus,

$$
0=\int_{r}^{s} S=\int_{r}^{s} T=\int_{r}^{s} U=\int_{r}^{s} V
$$

for $a<r<s<x$. Also, each of $S, T, U$ and $V$ has bounded variation on [ $a$, $x]$. Thus, it follows from Lemma 1 that there exists a subdivision $D$ of $[a, x]$ such that, if $\left\{x_{i}\right\}_{i=0}^{n}$ is a refinement of $D$, then each of the expressions in (a), (b), (c) and (d) is less than $\varepsilon / 4$. Hence, if $\left\{x_{i}\right\}_{i=0}^{n}$ is a refinement of $D$, then

$$
\begin{gathered}
\left|f(x)-\left\{w(x)+\sum_{i=1}^{n}\left(f_{i-1} H_{i}+G_{i} f_{i}+f_{i-1} K_{i} f_{i}\right)\right\}\right| \\
<\varepsilon / 4+\varepsilon / 4+\varepsilon / 4+\varepsilon / 4=\varepsilon .
\end{gathered}
$$

Hence, $(L R L R) \int_{a}^{x}(f H+G f+f K f)$ exists and is $f(x)-w(x)$. Therefore, (2) implies (1). This completes the proof of Theorem 4.

\section{BIBLIOGRAPHY}

1. D. P. Puig Adam, Los fracciones continuaus de cocientes incompletos differenciales y sus aplicaciones, Revista Mat. Hisp.-Amer. (4) 11 (1951), 180-190. (Spanish) MR 13, 540.

2. W. D. L. Appling, Interval functions and real Hilbert spaces, Rend. Circ. Mat. Palermo (2) 11 (1962), 154-156. MR 27 \#4040.

3. C. W. Bitzer, Stieltjes-Volterra integral equations, Illinois J. Math. 14 (1970), 434-451.

4. - Convolution, fixed point, and approximation in Stieltjes-Volterra integral equations, J. Austral. Math. Soc. 14 (1972), 182-199. MR 47 \#5528.

5. J. R. Dorroh, Integral equations in normed abelian groups, Pacific J. Math. 13 (1963), 1143-1158. MR 28 \# 1469. 
6. F. R. Gantmacher, The theory of matrices, Vol. 2, GITTL, Moscow, 1953; Chelsea, New York, 1959. MR 21 \#6372c.

7. B. W. Helton, Integral equations and product integrals, Pacific J. Math. 16 (1966), 297-322. MR 32 \#6167.

8. , A product integral representation for a Gronwall inequality, Proc. Amer. Math. Soc. 23 (1969), 493-500. MR 40 \#1562.

9. , Solutions of $f(x)=f(a)+(R L) \int_{a}^{x}(f H+f G)$ for rings, Proc. Amer. Math. Soc. 25 (1970), 735-742. MR 41 \# 4159.

10. , The solution of a nonlinear Gronwall inequality, Proc. Amer. Math. Soc. 38 (1973), 337-342. MR 46 \#9287.

11. _ A product integral solution of a Riccati equation, Pacific J. Math. 56 (1975), 113-130.

12. _ A special integral and a Gronwall inequality, Trans. Amer. Math. Soc. 217 (1976), 163-181.

13. J. C. Helton, An existence theorem for sum and product integrals, Proc. Amer. Math. Soc. 39 (1973), 149-154. MR 47 \#5596.

14. , Mutual existence of sum and product integrals, Pacific J. Math. 56 (1975), $495-516$.

15. , Product integrals and the solution of integral equations, Pacific J. Math. 58 (1975), 87-103.

16. , Solution of integral equations by product integration, Proc. Amer. Math. Soc. 49 (1975), 401-406.

17. _ Mutual existence of product integrals in normed rings, Trans. Amer. Math. Soc. 211 (1975), 353-363.

18. J. V. Herod, Solving integral equations by iteration, Duke Math. J. 34 (1967), 519-534. MR 36 \#625.

19. , Multiplicative inverses of solutions for Volterra-Stieltjes integral equations, Proc. Amer. Math. Soc. 22 (1969), 650-656.

20. , A pairing of a class of evolution systems with a class of generators, Trans. Amer. Math. Soc. 157 (1971), 247-260. MR 43 \#6778.

21. - A product integral representation for an evolution system, Proc. Amer. Math. Soc. 27 (1971), 549-556. MR 43 \# 1001.

22. T. H. Hildebrandt, On systems of linear differentio-Stielties-integral equations, Illinois J. Math. 3 (1959), 352-373. MR 21 \#4339.

23. D. B. Hinton, A Stieltjes-Volterra integral equation theory, Canad. J. Math. 18 (1966), 314-331. MR 32 \#6169.

24. D. L. Lovelady, Addition in a class of nonlinear Stieltjes integrators, Israel J. Math. 10 (1971), 391-396. MR 45 \# 7548.

25. , Algebraic structure for a set of nonlinear integral operations, Pacific J. Math. 37 (1971), 421-427. MR 46 \#2485.

26. , Multiplicative integration of infinite products, Canad. J. Math. 23 (1971), 692-698. MR 44 \#4539.

27. , Perturbations of solutions of Stieltjes integral equations, Trans. Amer. Math. Soc. 155 (1971), 175-187.

28. , Product integrals for an ordinary differential equation in a Banach space, Pacific $\mathrm{J}$. Math. 48 (1973), 163-168. MR 49 \#3283.

29. J. S. Mac Nerney, Stieltjes integrals in linear spaces, Ann. of Math. (2) 61 (1955), 354-367. MR 16, 716.

30. , Continuous products in linear spaces, J. Elisha Mitchell Sci. Soc. 71 (1955), 185-200. MR 18, 54.

31. , Determinants of harmonic matrices, Proc. Amer. Math. Soc. 7 (1956), 1044-1046. MR 18, 906.

32. , Integral equations and semigroups, Illinois J. Math. 7 (1963), 148-173. MR 26 \#1726. 
33. \#4133.

34. __ A nonlinear integral operation, Illinois J. Math. 8 (1964), 621-638. MR 29 \# 5082.

35. P. R. Masani, Multiplicative Riemann integration in normed rings, Trans. Amer. Math. Soc. 61 (1947), 147-192. MR 8, 321.

36. J. W. Neuberger, Continuous products and nonlinear integral equations, Pacific J. Math. 8 (1958), 529-549. MR 21 \# 1509.

37. __ Concerning boundary value problems, Pacific J. Math. 10 (1960), 1385-1392. MR 23 \#A2012.

38. __ A generator for a set of functions, Illinois J. Math. 9 (1965), 31-39. MR 30 \#2369.

39. J. A. Reneke, A product integral solution of a Stieltjes-Volterra integral equation, Proc. Amer. Math. Soc. 24 (1970), 621-626. MR 40 \#6214.

40. , Product integral solutions for hereditary systems, Trans. Amer. Math. Soc. 181 (1973), 483-493. MR 48 \#2692.

41. , Stieltjes integral equations in partially ordered sets, J. Math. Anal. Appl. 50 (1975), 288-302.

42. H. S. Wall, Concerning continuous continued fractions and certain systems of Stieltjes integral equations, Rend. Circ. Mat. Palermo (2) 2 (1953), 73-84. MR 15, 533.

43. , Concerning harmonic matrices, Arch. Math. 5 (1954), 160-167. MR 15, 801.

44. G. F. Webb, Nonlinear evolution equations and product integration in Banach spaces, Trans. Amer. Math. Soc. 148 (1970), 273-282. MR 42 \#901.

45. , Product integral representation of time dependent non-linear evolution equations in Banach spaces, Pacific J. Math. 32 (1970), 269-281. MR 41 \#2483.

46. - Nonlinear evolution equations and product stable operators on Banach spaces, Trans. Amer. Math. Soc. 155 (1971), 409-426. MR 34 \#2582.

Department of Mathematics, Arizona State University, Tempe, Arizona 85281 